\section{Tratamiento ortodóncico de un paciente Clase III con pérdida de inserción periodontal, agenesia y transposición dental. Reporte de un caso}

\section{Orthodontic treatment of a Class III patient with periodontal attachment loss, agenesis and dental transposition. A case report}

\section{Resumen}

Un aspecto fundamental en el tratamiento de la ortodoncia es el uso correcto de la biomecánica. Esto adquiere mayor relevancia en pacientes que presentan pérdida de inserción periodontal y más aún si añadimos problemas dentales como agenesia y transposición dentaria. El presente artículo reporta el caso de un paciente femenino de 24 ańos, que presentaba una maloclusión de Clase III y se logró corregir obteniendo una oclusión estable y funcional, así como la disminución del trauma oclusal inicial. Adicionalmente se mejoró la estética de los dientes en todo el sector anterosuperior debido al desbalance dejado por la agenesia del incisivo central y la transposición del canino.

Palabras clave: Agenesia dental; Clase III de Angle; Ortodoncia; Periodoncio; Tratamiento.

\begin{abstract}
A fundamental aspect in the treatment of orthodontics is the correct use of biomechanics; especially in patients who present loss of periodontal insertion and conditions such as agenesis and dental transposition. This article reports the treatment of a 24-year-old female patient, who presented a Class III malocclusion; which was corrected obtaining a stable and functional occlusion, as well as the reduction of the initial occlusal trauma. Additionally, the aesthetics was improved throughout the antero-superior sector; which was caused by the imbalance left by the central incisor's agenesis and the transposition of the canine.
\end{abstract}

Keywords: Anodontia; Angle class III; Orthodontics; Periodontium; Treatment.

\section{Caso Clínico}

Giancarlo Alfaro Ramos 1,a, Hugo Javier Luque Luque ${ }^{1, b}$

${ }^{1}$ Facultad de Odontología. Universidad Nacional Mayor de San Marcos. Lima, Perú.

${ }^{a}$ Cirujano Dentista

${ }^{\mathrm{b}}$ Especialista en Ortodoncia y Ortopedia Maxilar

Correspondencia:

Giancarlo Alfaro Ramos

Correo electrónico: giancarlo_alfaro@hotmail. com

Jirón Mariano Melgar 525, Carmen de La Legua. Callao, Perú.

\section{Coautor: \\ Hugo Javier Luque Luque} hjll50@hotmail.com

Conflicto de intereses: Los autores declaran no tener conflictos de interés.

Fuente de financiamiento: Autofinanciado

Fecha de recepción: 22/09/17

Fecha de aceptación: 04/12/17 


\section{Introducción}

La relación esquelética y maloclusión de Clase III ${ }^{1}$ se considera como uno de los problemas más difíciles de tratar debido a un prognatismo mandibular, un maxilar deficiente o una combinación de ambas. Esto en algunos casos conlleva a asimetrías faciales y problemas funcionales que repercuten en la oclusión provocando trauma oclusal, periodonto reducido, recesiones gingivales y movilidad dental ${ }^{2-5}$. La decisión de camuflar o tratarla de manera quirúrgica es viable. La opción de un tratamiento de camuflaje ortodóncico se da en pacientes que presentan discrepancia esqueletal de Clase III $^{2}$ donde se puede realizar la protrusión e inclinación de los incisivos superiores y retroinclinación de los incisivos inferiores, así como el incremento del tercio inferior con una rotación horaria de la mandíbula ${ }^{6}$. Las anomalías dentales como la agenesia y transposición son más prevalentes en el sexo femenino ${ }^{7}$. La transposición de una pieza dental se define como el cambio de posición de dos piezas adyacentes en el mismo cuadrante, siendo más frecuente en la maxila que la mandíbula y los dientes más afectados son los caninos ${ }^{8-11}$.

La transposición puede ser clasificada como completa o incompleta. Se considera una transposición completa cuando tanto las coronas y las raíces son halladas completamente en posición transpuesta, mientras que en la incompleta las coronas pueden estar transpuestas, pero las raíces permanecen en una posición normal ${ }^{12}$. Según estos criterios diagnósticos podemos determinar si mantenemos la transposición o si se realiza la corrección a su posición original, también se debe considerar la parte funcional y estética ${ }^{10,12,13}$.

Este reporte de caso presenta un tratamiento de camuflaje de Clase III usando diferentes biomecánicas con arco continuo, arco segmentado, arcos multiloops y elásticos intermaxilares.

\section{Reporte del caso}

Diagnóstico y etiología. Paciente femenino de 24 años 6 meses de edad acudió a la Clínica de Posgrado de la Facultad de Odontología de la Universidad Nacional Mayor de San Marcos. La paciente refirió: "Tengo mis dientes chuecos y quiero mejorar mi sonrisa”. Al análisis clínico extraoral presentó un biotipo mesofacial, un perfil total convexo, asimetría leve, protrusión labial en reposo, labio superior corto y sonrisa asimétrica (Figura 1).

$\mathrm{Al}$ análisis clínico intraoral y de modelos, se determinó un biotipo gingival delgado, y periodonto reducido a nivel del sector anterior, una maloclusión Clase III de Angle subdivisión izquierda, mordida cruzada anterior, mordida cruzada posterior del lado derecho, mordida abierta posterior del lado izquierdo, línea media superior e inferior no coincidente, apiñamiento anterosuperior y anteroinferior de $-5 \mathrm{~mm}$ y $-6 \mathrm{~mm}$ respectivamente, discrepancia de Bolton anterior por exceso inferior de $2 \mathrm{~mm}$, overbite y overjet disminuidos de -1 y $-2 \mathrm{~mm}$ respectivamente, ausencia de la pieza dentaria 4.4 , agenesia de pieza dentaria 1.1 y transposición de las piezas dentarias 1.4 con 1.3 , ausencia de la tercera molar inferior derecha (Figuras 1 y 2).

El análisis funcional presenta deglución adaptada y alteración en la fonética (ceceo).

El análisis de la radiografía panorámica mostró la ausencia de las piezas dentarias 1.1, 4.4 y 4.8, vías aéreas poco permeables, hipertrofia de cornetes inferiores, septum nasal desviado hacia la izquierda, altura y ancho de las ramas mandibulares simétricas, así como pérdida de la cortical vestibular de los dientes inferiores, esto se corroboró con la tomografía Cone Beam (Figuras 3 y 4).

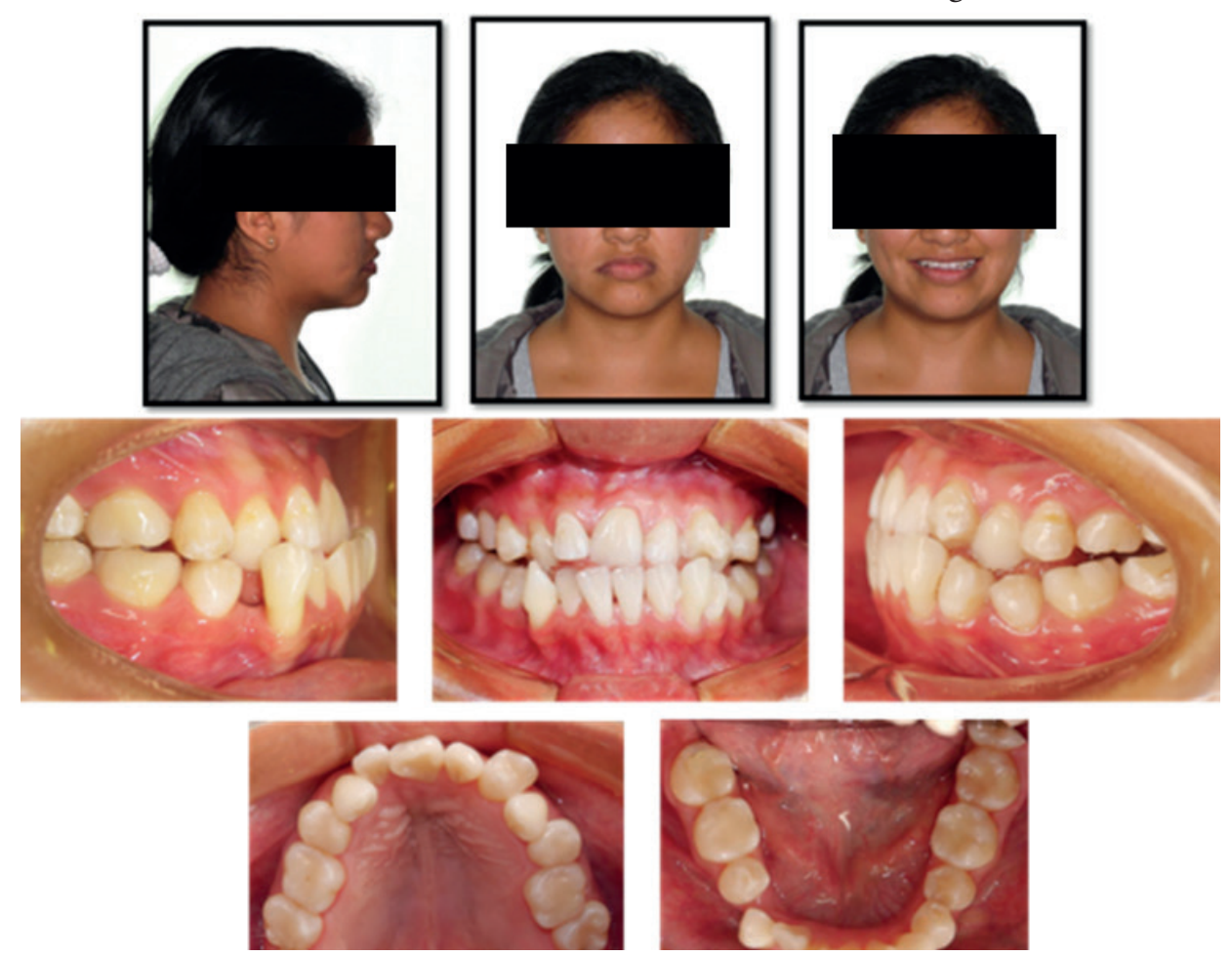

Figura 1. Fotografías extraorales e intraorales pretratamiento 

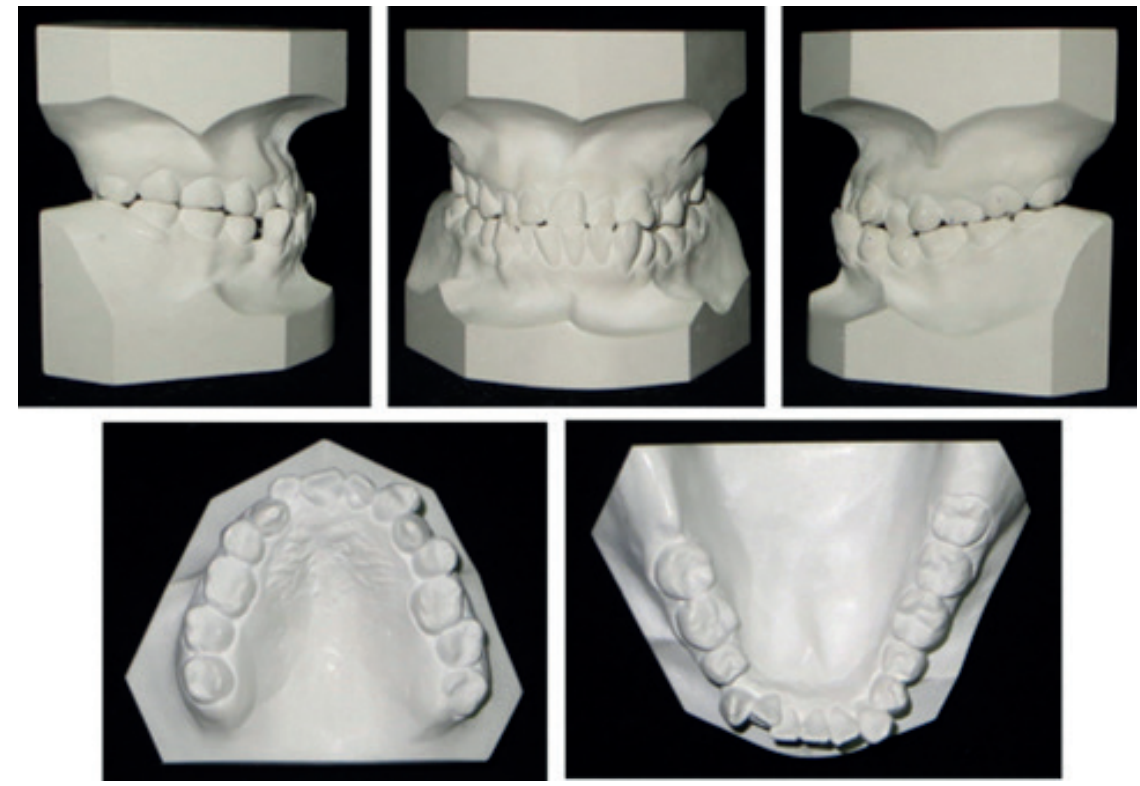

Figura 2. Modelos dentales pretratamiento

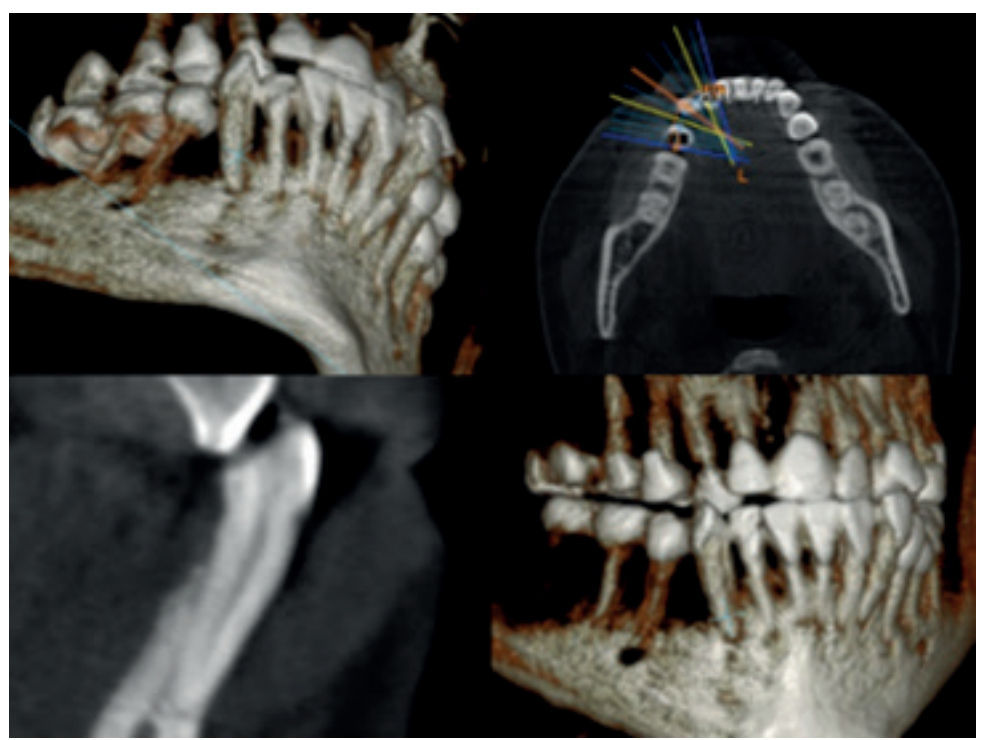

Figura 3. Tomografía Cone Beam para visualizar pérdida de inserción periodontal

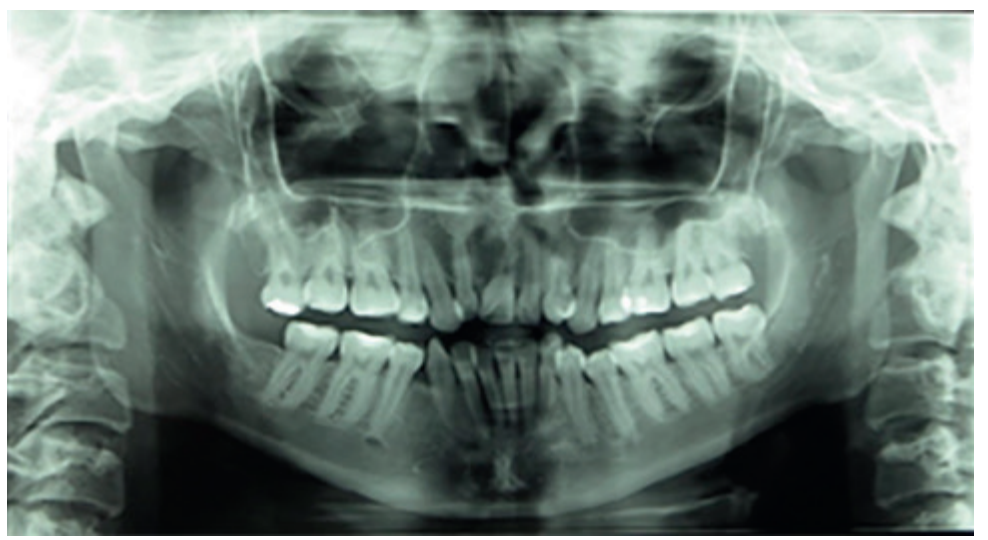

Figura 4. Radiografía panorámica pretratamiento 
El análisis cefalométrico de Steiner, Tweed y Downs indicó una relación esquelética de Clase III por retrusión maxilar, plano mandibular normodivergente, incisivos superior e inferior ligeramente vestibularizados y labio inferior protruido (Figura 5, Tabla 1). El índice de discrepancia según la ABO (American Board of Orthodontics), al inicio de tratamiento fue de 48 .

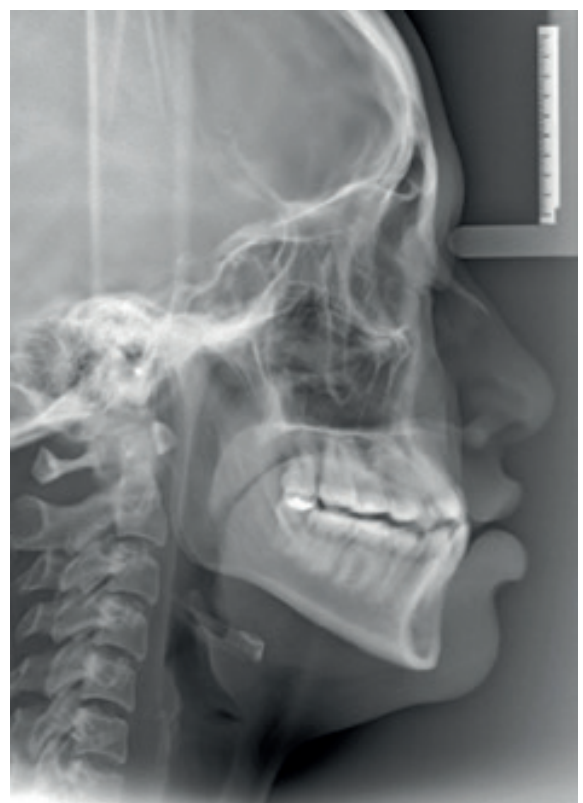

Figura 5. Radiografía cefalométrica pretratamiento

Tabla 1. Medidas cefalométricas pretratamiento y postratamiento

\begin{tabular}{lcc}
\hline & Pretratamiento & Postratamiento \\
\hline Angulo SNA $\left(^{\circ}\right)$ & 79 & 79 \\
Angulo SNB $\left(^{\circ}\right)$ & 80 & 80 \\
Angulo ANB $\left(^{\circ}\right)$ & -1 & -1 \\
Angulo SND $\left(^{\circ}\right)$ & 76 & 76 \\
1_NA $(\mathrm{mm})$ & 6 & 8 \\
1:NA $\left(^{\circ}\right)$ & 30 & 32 \\
1_NB $(\mathrm{mm})$ & 12 & 3 \\
1:NB $\left(^{\circ}\right)$ & 20 & 23 \\
Pog_NB $(\mathrm{mm})$ & 0 & 0 \\
IMPA $\left({ }^{\circ}\right)$ & 93 & 84 \\
FMA $\left(^{\circ}\right)$ & 28 & 26 \\
1:1 $\left({ }^{\circ}\right)$ & 120 & 128 \\
Ocl:SN $\left({ }^{\circ}\right)$ & 16 & 28 \\
GoGn:SN $\left({ }^{\circ}\right)$ & 35 & 36 \\
Labio superior: línea S $(\mathrm{mm})$ & 0 & 1 \\
Labio inferior: línea S $(\mathrm{mm})$ & 5 & 4 \\
Eje Y $\left(^{\circ}\right)$ & 64 & 61 \\
\hline
\end{tabular}

Objetivos del tratamento. Los objetivos del tratamiento basados en lograr una oclusión estable, así como restaurar la estética, función y salud periodontal de la región anterior fueron: mantener el perfil de los labios, corregir la maloclusión de Angle Clase III, la mordida cruzada anterior y posterior, corregir el overbite y overjet, eliminar el apiñamiento superior e inferior, eliminar el exceso anteroinferior de Bolton, conseguir una ade- cuada relación funcional anterior y una desoclusión canina derecha e izquierda, mimetizar las piezas anteriores producto de la agenesia y transposición dental.

Alternativas del tratamiento. La primera opción fue un tratamiento ortoquirúrgico para adelantar la maxila y retroceder la mandíbula combinado con el tratamiento ortodóncico fijo, esto conseguiría una mejora en el perfil facial; se le planteó a la paciente, pero se rehusó debido a factores económicos, así como los riesgos quirúrgicos que conllevaría.

La segunda opción en el maxilar fue crear el espacio para un implante de la pieza dentaria 1.1, debido a la agenesia que presentaba la paciente, así como la exodoncia de la pieza dentaria 2.4, para crear el espacio necesario para el incisivo central y la corrección de la línea media. En la mandíbula, exodoncia de la pieza dentaria 3.4 para conseguir descruzar la mordida anterior y buscar un camullaje dentario.

Como tercera opción, se planteó que la pieza dentaria 1.1 sea reemplazada por la pieza dentaria 2.1 y la pieza dentaria 2.2 asuma la posición de la pieza dentaria 2.1, mantener la transposición de las piezas dentarias 2.3/2.4. En la arcada inferior, se realizaría la extracción de la pieza dentarias 3.4 para corregir el apiñamiento, el desvió de la línea media y la mordida cruzada anterior. Después de considerar todas las opciones y consultado con el paciente, la tercera opción fue la elegida.

Progreso del tratamiento. Se instaló un sistema de brackets edgewise standard slot 0,022". El primer mes de tratamiento de ortodoncia, todas las terceras molares fueron extraídas y se realizó la extracción de la pieza dentaria 3.4.

En la fase de alineación se colocó en la arcada superior un arco Niti 0,014" termoactivado para mejorar el perímetro. Una semana después de la extracción, se colocó en la arcada inferior un arco Niti termoactivado 0,014" cinchado y con un laceback a nivel de canino.

En el siguiente control se incluyeron las bandas en los segundos molares tanto en la arcada superior e inferior, se siguió el proceso de alineamiento con arco de Niti termoactivado 0,016 ", en la arcada inferior siempre cinchado y laceback a nivel de canino (Figura 6).

En los controles siguientes, se buscó la nivelación así como el cierre de espacio y corrección de la línea media; para tal fin se empleó en la arcada inferior un splint de mordida de acrílico unido por un alambre de acero 1,0. Se colocó en la arcada inferior un arco de cierre de espacios de acero 0,016”. Teniendo en consideración que el paciente tenía periodonto reducido, todas las fuerzas aplicadas fueron medidas usando un gramómetro. Se usaron auxiliares segmentados para la corrección de la línea media tanto superior como inferior. Se consiguió el espacio suficiente para mimetizar la pieza dentaria 2.2 , se realizó una ameloplastía positiva de corona para mejorar su anatomía y convertirla en el incisivo central superior izquierdo, con el sistema de resina universal Filtek Z350 XT, se realizaron desgastes interproximales a la pieza dentaria 2.4 para simular el tamaño del incisivo lateral superior izquierdo, 
también se realizaron ameloplastía negativa (desgastes) de manera secuencial de la cúspide palatina para evitar las interferencias al momento de la protrusiva.

Para distalizar la pieza dentaria 3.3 se usó un ansa en $\mathrm{T}$ de Burstone como primera opción. En la arcada superior, se colocó un arco de acero 0,017 " x 0,025 ”; mientras que en la arcada inferior, una vez conseguido el cierre de espacio, se retiró el splint de mordida y se colocó un arco multiloop 0,016" para nivelar los dientes, seguido después por arcos continuos 0,016 " y 0,018 " con dobleces de primer orden. Se prosiguió en la arcada superior como inferior con arcos de acero 0,017"x 0,025 " y dobleces de primer orden para compensar el ancho vestibulo-palatino. Luego de 24 meses, se consiguió cerrar el espacio en la arcada inferior y corregir la mordida cruzada anterior (Figura 6).

Se prosiguió después a la fase de correcciones sagitales, verticales y transversales como etapa de finalización, que inicio con la colocación de dos arcos multiloop edgewise arch wire (MEAW) Elgiloy azul de 0,017"x 0,025 " tanto en la arcada superior como inferior con el objetivo de conseguir un buen engranaje posterior y una oclusión funcional. En una primera etapa, el arco MEAW actuó junto con un arco Overlay para mejorar la parte transversal posterior superior, luego se usaron elásticos intermaxilares asimétricos de clase III izquierdo y clase II derecho de 3/16 x 6.5onz. Después de 4 meses se regresó a arcos continuos superior e inferior en acero inoxidable de 0,018 "x 0,025 " con dobleces de primer y segundo orden para nivelar crestas y surcos principales. Después de un mes en la arcada superior se segmentó el arco de acero 0,018 "x 0,025 ” y en la arcada inferior se colocó un arco de acero 0,016 " se usaron elásticos verticales de 1/8 3,5 Onz. Se realizaron desgastes selectivos para mejorar el overjet y overbite y la intercuspidación (Figura 7).

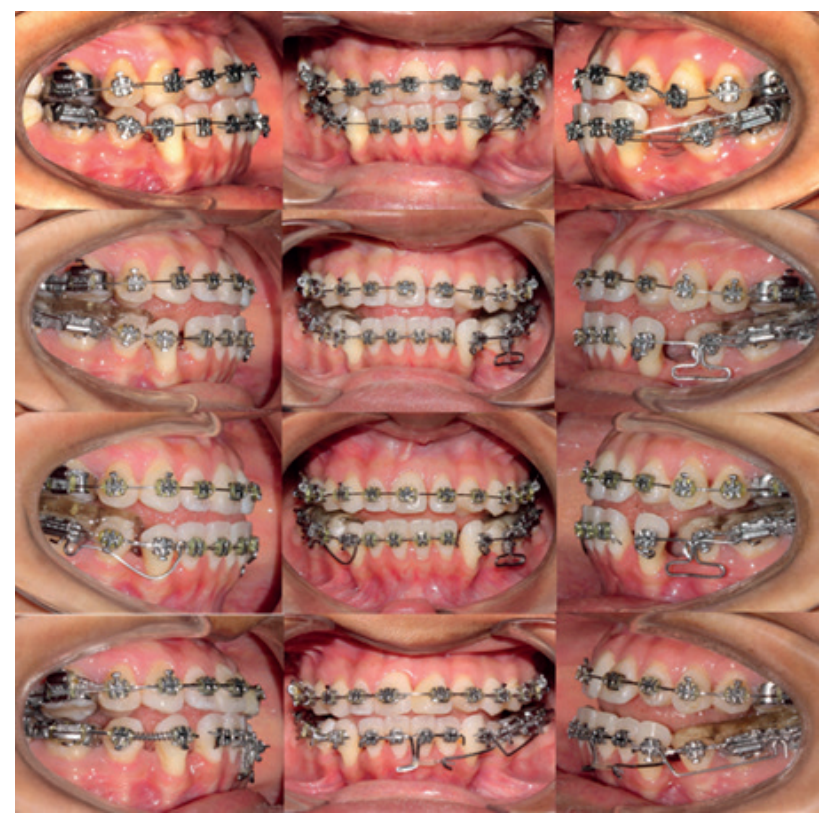

Figura 6. Fotografías intraorales durante el tratamiento. Alineamiento y nivelación y cierre de espacios

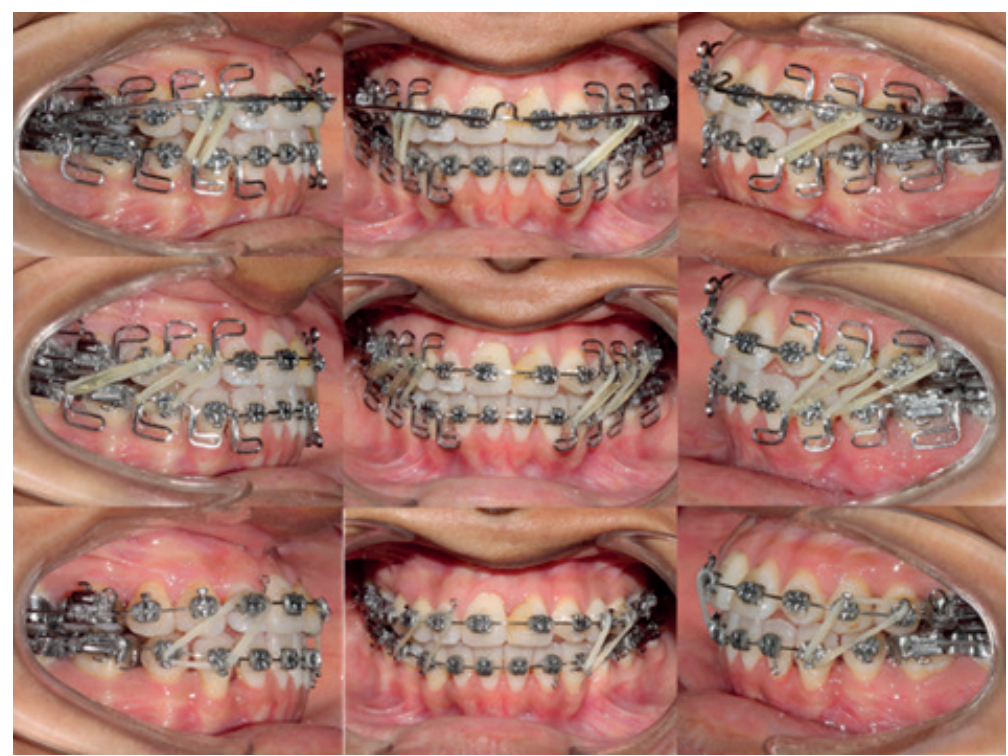

Figura 7. Fotografías intraorales durante el tratamiento. Finalización 
Cuando se retiró la aparatología fija superior e inferior, se procedió al pulido de todos los dientes y se tomaron impresiones para realizar los aparatos de contención, los cuales fueron en la arcada superior mixta con contención fija de canino a canino con alambre bond a braid y placa Hawley removible; mientras que en la arcada inferior, una contención fija con alambre de acero 0,028 " de segunda premolar a segunda premolar (Figura 8).

Resultados del tratamiento. Durante los 32 meses de tratamiento, se consiguieron los objetivos trazados, facial- mente no hubo cambios muy significativos, pero sí en el tercio inferior (Figura 8).

En los registros fotográficos y de modelos se observa relaciones molares, premolares y caninos aceptables, se corrigió la mordida cruzada anterior y posterior, se consiguieron el overbite y overjet adecuados, la línea media dentaria centrada con la línea media facial, se eliminó las discrepancias dentoalveolar y de Bolton (Figuras 8 y 9 ).
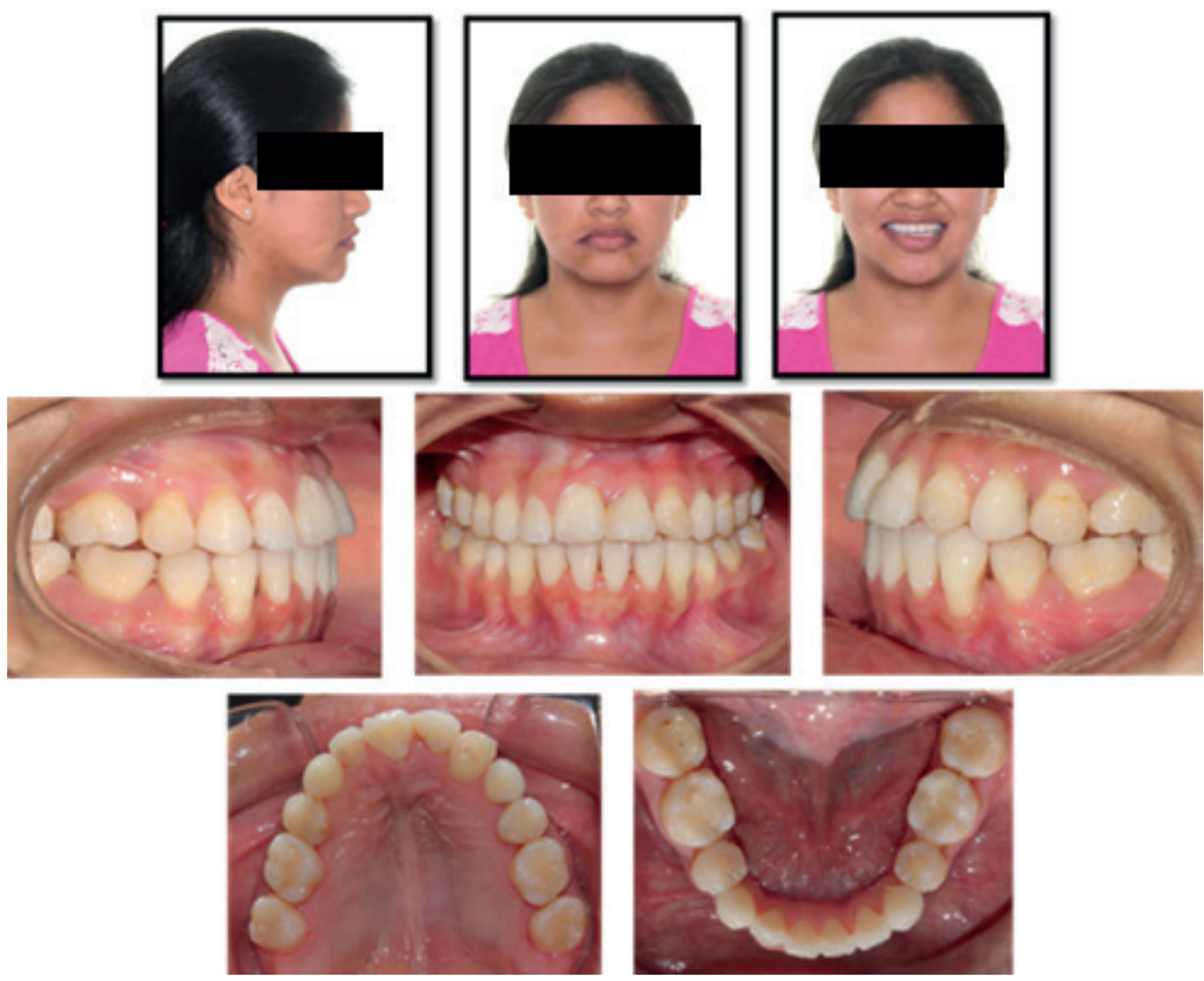

Figura 8. Fotografías extraorales e intraorales postratamiento
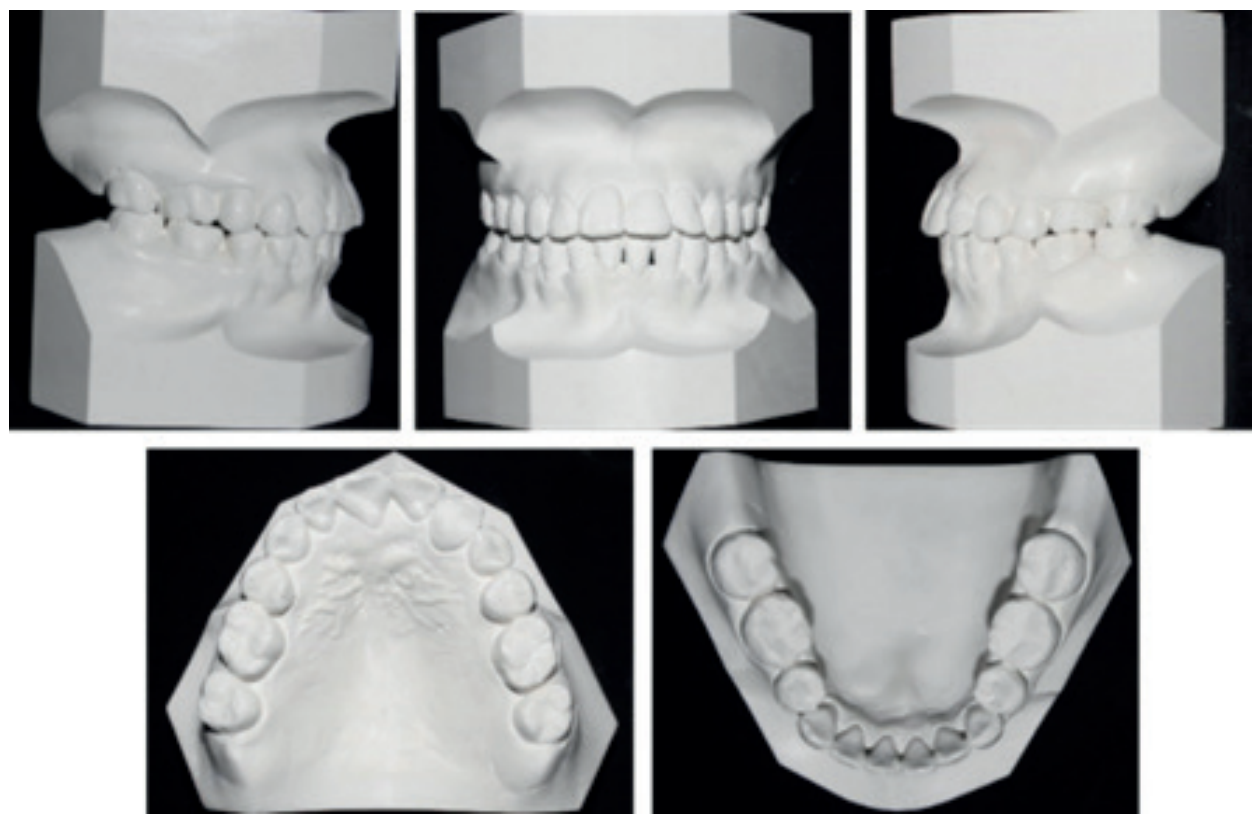

Figura 9. Modelos dentales postratamiento 
Se consiguió relaciones funcionales en la protrusiva y lateralidades derecha e izquierda, no se reportó ninguna alteración de la articulación temporomandibular antes, durante y después del tratamiento ortodóncico.

La radiografía panorámica de post tratamiento, no mostró significativa reabsorción radicular ni ósea. Se observó paralelismo radicular en la mayoría de piezas (Figura 10).

El análisis cefalométrico final así como las superposiciones, no mostraron cambios a nivel de bases óseas o relaciones esqueléticas, pero si mostraron cambios en el tercio inferior, posición de labios, plano oclusal y posición de los incisivos (Figuras 11, 12 y Tabla 1).
El índice de discrepancia de la $\mathrm{ABO}$ al final de tratamiento tuvo un puntaje de 11 , lo cual indica un adecuado tratamiento.

A los 6 meses después del retiro de la aparatología, se evidenció la estabilidad de los resultados obtenidos durante el tratamiento, buenas relaciones intraarco e interarco, buen funcionamiento de las guías anterior y lateralidad. Para mimetizar la agenesia y la transposición en el sector antero superior, se realizó una interconsulta con el área de Odontología Restauradora y Estética, se evaluó y planificó el uso del diseño digital de sonrisa y un Mock Up. Se logró una armonía estética en el sector antero superior, mediante la colocación de carillas de resinas de canino a canino (Figura 13 y 14).

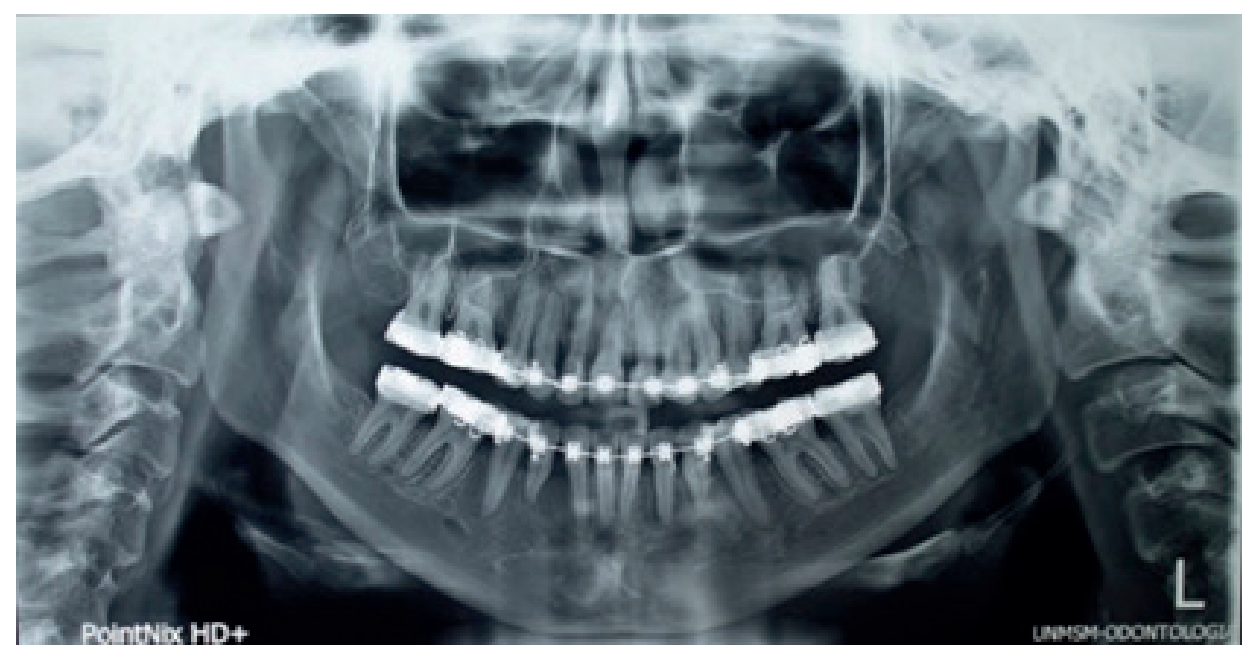

Figura 10. Radiografía panorámica postratamiento

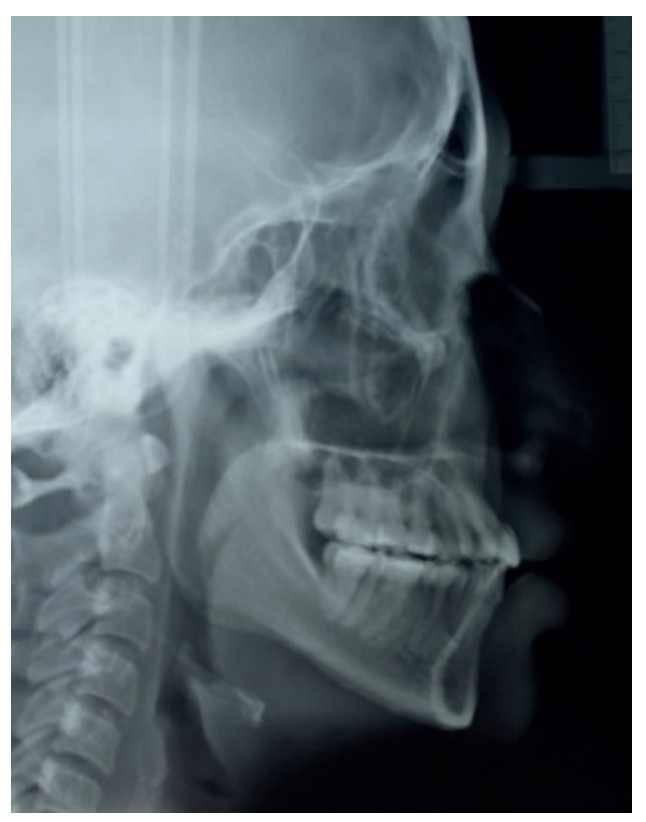

Figura 11. Radiografía cefalométrica postratamiento

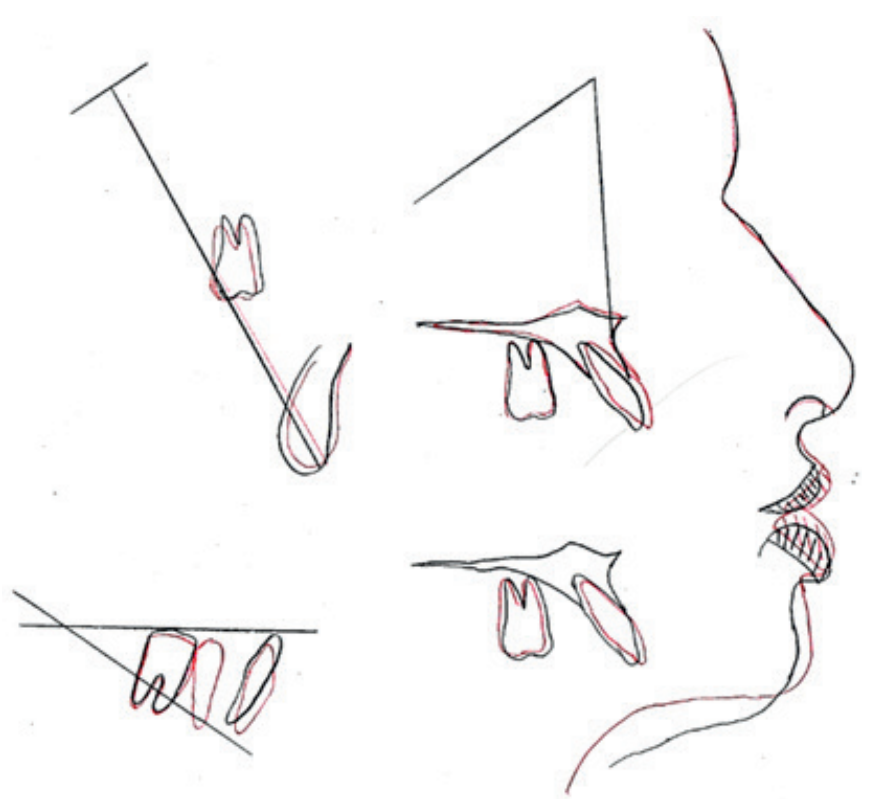

Figura 12. Superposiciones de trazados cefalométricos de pretratamiento (línea de color negro) y de postratamiento (línea de color rojo). Según los cinco campos de Ricketts 

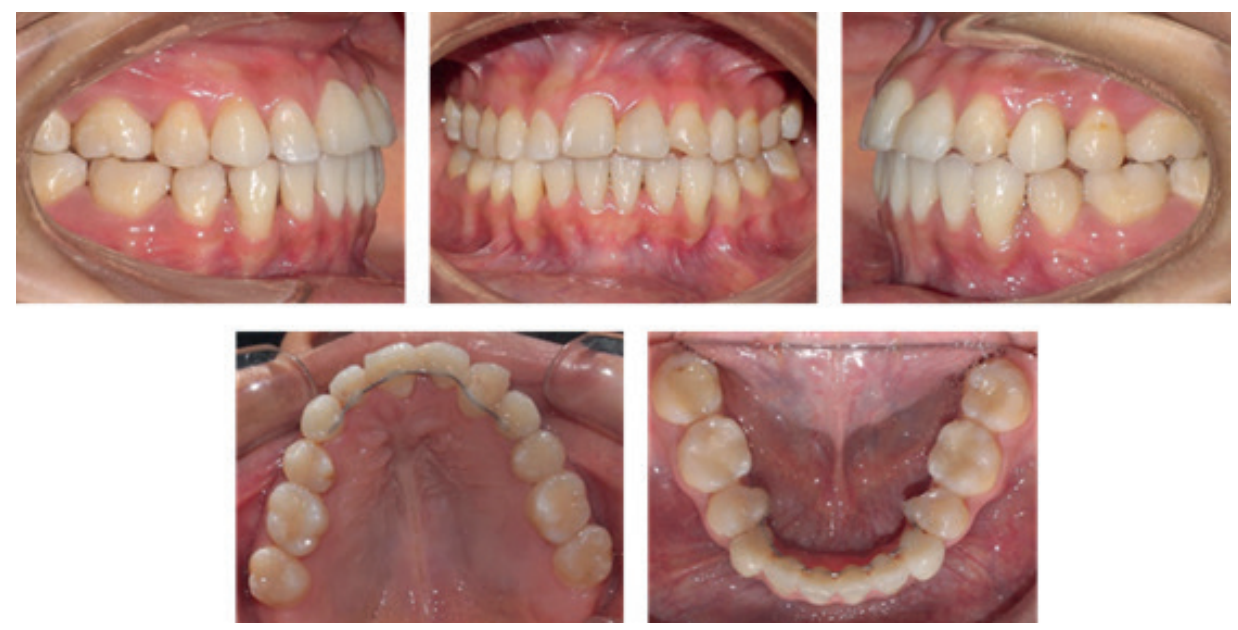

Figura 13. Fotografías intraorales 6 meses postratamiento

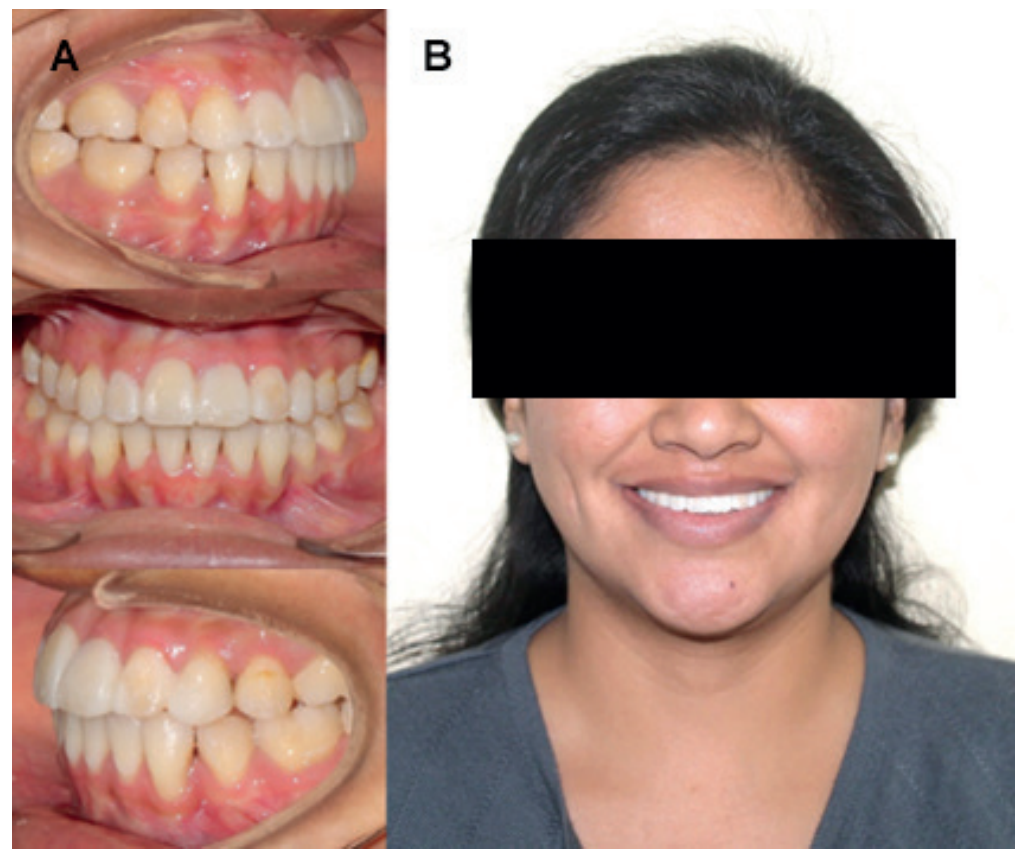

Figura 14. A. Fotografía de mock up, después del diseño de sonrisa, B. Foto de sonrisa con carilla de resina directa

\section{Discusión}

El mejor momento para corregir una maloclusión de Clase III es la dentición mixta, antes de los 8 a 10 años de edad mediante el tratamiento ortopédico maxilar ${ }^{14,15}$; sin embargo cuando estamos en una dentición permanente y el paciente dejó de crecer se realiza el camuflaje ortodóncico. Moon et al. ${ }^{16}$ señalan que los pacientes de Clase III deben de tener características ideales para que respondan mejor al tratamiento de camuflaje.

En cuanto a los problemas dentales que presenta la paciente, se ha descrito en la literatura opciones sin extracciones dentales para casos de transposición dentarias completas. Si bien existen reportes sobre el manejo y corrección de la transposición ${ }^{4}$, es el profesional quien determina la biomecánica para cumplir con los objetivos planteados.

En el caso presentado, la recesión y biotipo gingival eran desfavorables; por ello se tomó la decisión de realizar movimientos dentarios sin generar mayor perjuicio y en un periodo que permita la reposición de las piezas dentarias evitando dañar más las estructuras de soporte 10,12,13. En el maxilar superior se optó por no extraer una pieza dentaria, por las condiciones desfavorables que se encontró inicialmente, la mejor opción debido a todas las características negativas fue buscar aumentar el volumen del arco y mimetizar las piezas dentarias presentes para poder reemplazar la agenesia y la transposición; al dar un mejor perímetro de arco, se pudo corregir la mordida cruzada anterior. La decisión de extraer una pieza dentaria más en el maxilar inferior fue el de buscar simetría y corregir la mordida cruzada anterior, pero se tenía que tener una biomecánica adecuada a las condiciones periodontales presentes en el maxilar inferior, debido a este problema, las fuerzas que se emplearon fueron leves y continuas, para evitar mayores efectos indeseados en los dientes con menor soporte periodontal, las fuerzas fueron medidas en cada cita para disminuir efectos adversos ${ }^{17}$. 
La biomecánica con arcos segmentados, arcos multiloop y arco continuo fueron aplicados secuencialmente en cada etapa del tratamiento con el fin de mejorar las relaciones sagitales, transversales y verticales de la oclusión ${ }^{18}$.

Se concluye que el camuflaje ortodóncico es una opción terapéutica que se puede realizar en pacientes que tengan características favorables como por ejemplo posición e inclinación de incisivos, hipodivergencia del plano mandibular y tercio inferior disminuido. En pacientes con pérdida de inserción periodontal, la biomecánica debe ser la más eficiente con uso de fuerzas constantes y ligeras para evitar la recesión gingival y reabsorción radicular.

La biomecánica con arco segmentado, arco multiloop y arco continuo pueden ser aplicados en diferentes fases de tratamiento y permite mayor versatilidad y posibilidad terapéutica para casos complejos.

\section{Referencias bibliográficas}

1. Angle E. Classification of malocclusion. Dental Cosmos. 1899;41:248-64.

2. Kama JD, Ozer T, Baran S. Orthodontic and orthopaedic changes associated with treatment in subjects with Class III malocclusions. Eur J Orthod. 2006;28(5):496502.

3. Chew MT. Soft and hard tissue changes after bimaxillary surgery in Chinese Class III patients. Angle Orthod. 2005;75(6):959-63.

4. Matheus D, Kokich V. Managing treatment for the orthodontic patient with periodontal problems. Semin Orthod.1997 Mar; 3(1):21-38.

5. Baccetti T, Reyes BV. Gender differences in Class III malocclusion. Angle Orthod. 2005;73(2):136-45.

6. Lin J, Gu Y. Preliminary investigation of nonsurgical treatment of severe skeletal Class III malocclusion in the permanent dentition. Angle Orthod. 2003;73(4):40110.
7. Polder BJ, Van't Hof MA. A meta-analysis of the prevalence of dental agenesis of permanente teeth. Commun Dent Oral Epidemiol. 2004;32(3):217-26.

8. Peck S, Peck L. Classification of maxillary tooth transpositions. Am J Orthod Dentofacial Orthop. 1995;107(5):505-17.

9. Shapira Y, Kuftinec MM. Orthodontic management of mandibular canine - incisor transposition. Am J Orthod 1983;83(4):271-6.

10. Maia FA, Maia NG. Unusual Orthodontic Correction of Bilateral Maxillary Canine-First Premolar Transposition. Angle Orthod. 2005;75(2):266-76.

11. Papadopoulos MA, Chatzoudi M. Prevalence of tooth transposition: a meta-analysis. Angle Orthod. 2010;80(2):275-85.

12. Giacomet F, Araújo MT. Orthodontic correction of a maxillary canine-first premolar transposition. Am J Orthod Dentofacial Orthop. 2009;136(1):115-23.

13. Capelozza L, De Almeida M, Li An Tien, Bertoz FA. Maxillary Canine - First premolar transposition: Restoring normal tooth order with segmented mechanics. Angle Orthodontist. 2007;77(1):167-75.

14. Kim JH, Viana MA, Graber TM, Omerza FF, BeGole EA. The effectiveness of protraction face mask therapy: a meta-analysis. Am J Orthod Dentofacial Orthop. 1999;115(6):675-85.

15. Kook YA, Bayome M, Park JH. New approach of maxillary protraction using modified C- palatal plates in Class III patients. Korean J Orthod. 2015;45(4):209-14.

16. Moon YM, Ahm SJ. Cephalometric predictors of long term stability in the early treatment of Class III malocclusion. Angle Orthod. 2005;75(5):747-53.

17. Reyes BC, Baccetti T. An estimate of craneofacial growth in Class III maloclusion. Angle Orthd. 2006;76(4):57784.

18. Sadao S. Orthodontic Therapy using Multiloop Edgewise Archwire. Bull Kanagawa Dent Coll. 2001;11(1):154156. 
